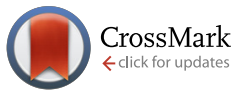

Cite this: Med. Chem. Commun., $2015,6,1761$

Received 6th June 2015,

Accepted 4th August 2015

DOI: $10.1039 / \mathrm{c} 5 \mathrm{md} 00241 \mathrm{a}$

www.rsc.org/medchemcomm

\section{Discovery of high affinity inhibitors of Leishmania donovani $\mathbf{N}$-myristoyltransferase $\dagger$}

\author{
Mark D. Rackham, \$\$ Zhiyong Yu, \$q James A. Brannigan, ${ }^{a}$ William P. Heal, $\|^{a}$ \\ Daniel Paape, ${ }^{\star \star c}$ K. Victoria Barker,$\dagger^{\mathrm{C}}{ }^{\mathrm{a}}$ Anthony J. Wilkinson, ${ }^{\mathrm{b}}$ Deborah F. Smith, ${ }^{\mathrm{c}}$ \\ Robin J. Leatherbarrowtta and Edward W. Tate*a
}

$\mathrm{N}$-Myristoyltransferase (NMT) is a potential drug target in Leishmania parasites. Scaffold-hopping from published inhibitors yielded the serendipitous discovery of a chemotype selective for Leishmania donovani NMT; development led to high affinity inhibitors with excellent ligand efficiency. The binding mode was characterised by crystallography and provides a structural rationale for selectivity.

\section{Introduction}

Leishmaniases are a spectrum of neglected tropical diseases resulting from the infection with parasites of the genus Leishmania spp., transmitted by the bite of the female Phlebotomine sandfly to the human host. The disease manifests in one of three forms (cutaneous, mucocutaneous and visceral $)^{1}$ and is responsible for an estimated 60000 deaths and in excess of 500000 new cases per annum. ${ }^{2}$ The majority of fatalities stem from visceral leishmaniasis, which is predominately the result of infection by Leishmania donovani (Ld). ${ }^{3}$ As with many neglected diseases, the leishmaniases are most prevalent in developing and newly industrialized nations ${ }^{4}$ and although treatments exist, the majority suffer from significant levels of resistance and toxicity. ${ }^{5}$ Leishmania spp. infections remain challenging to treat chemotherapeutically;

\footnotetext{
${ }^{a}$ Department of Chemistry, Imperial College London, South Kensington Campus, London, SW7 2AZ, UK. E-mail: e.tate@imperial.ac.uk; Tel: +44 (o) 2075943752

${ }^{b}$ Structural Biology Laboratory, Department of Chemistry, University of York, York, YO10 5DD, UK

${ }^{c}$ Department of Biology, University of York, York, YO10 5DD, UK

$\dagger$ Electronic supplementary information (ESI) available. The coordinates and structure factor files have been deposited in the Protein Data Bank under the accession codes 5A27 and 5A28. See DOI: 10.1039/c5md00241a

$\$$ MDR and ZY contributed equally to the preparation of this manuscript.

$\S$ Current address: GlaxoSmithKline, Gunnels Wood Road, Stevenage, SG1 2NY, UK. I| Current address: International Discovery Service Unit, WuXi AppTec, Shanghai, 200131, China.

|| Current address: Department of Chemistry, Kings College London, London SE1 1UL, UK.

** Current address: Wellcome Trust Centre for Molecular Parasitology, Institute of Infection, Immunity and Inflammation, University of Glasgow, Glasgow, G12 8TA, UK.

$\dagger$ Current address: Dehns, Aspect House, 84-87 Queens Road, Brighton, BN1 3XE, UK.

\$t Current address: Liverpool John Moores University, Egerton Court, 2 Rodney Street, Liverpool, L1 2UA, UK.
}

many currently marketed drugs display $\mathrm{EC}_{50}$ values in the low $\mu \mathrm{M}$ range in in vitro models, ${ }^{5,6}$ orders of magnitude higher than the potency typically targeted in an anti-infective drug discovery project. In order to combat the continued evolution of the parasite in developing resistance, new chemotherapies functioning by novel mechanisms of action are essential.

$N$-Myristoyltransferase (NMT) is a ubiquitous eukaryotic enzyme responsible for the attachment of the long chain fatty acid myristate to the $\mathrm{N}$-terminus of substrate proteins. This modification is crucial in many biological processes, ${ }^{7-10}$ and NMT has been implicated as a highly promising drug target in fungal ${ }^{11}$ and parasitic infections, ${ }^{12-14}$ as recently demonstrated in human malaria parasites. ${ }^{15}$ In the specific context of leishmaniasis, NMT has been validated genetically in Leishmania major $(\mathrm{Lm}){ }^{16}$ and through chemical proteomic approaches in $\mathrm{Ld},{ }^{17}$ and selective small molecule inhibitors of this drug target are highly desirable.

\section{Results and discussion}

We have previously reported the discovery of LdNMT inhibitors by high-throughput screening, ${ }^{18-21}$ as well as the discovery of Plasmodium falciparum (Pf) and Plasmodium vivax (Pv) NMT inhibitors by both high-throughput screening ${ }^{22}$ and piggy-back/lead-hopping approaches. ${ }^{23-25}$ Throughout this work, all compounds were routinely screened against all three parasitic enzymes (LdNMT, PfNMT and PvNMT) and Homo sapiens (Hs) NMT, where selectivity over the human orthologue was desired to minimise any potential toxicity from modulating endogenous myristoylation. 2,3-Substituted benzo $[b]$ thiophenes were discovered as ligand-efficient inhibitors of PfNMT, which incidentally displayed selectivity over LdNMT (Fig. 1). ${ }^{25}$

During studies to reduce the molecular weight and lipophilicity of 1 in the further development of the series against 
<smiles>COc1cccc(COC(=O)c2sc3ccccc3c2OC2CCNCC2)c1</smiles>

$$
\begin{array}{rl}
\mathrm{K}_{\mathrm{i}}(\mu \mathrm{M}):^{a} & 0.83 \text { (PfNMT) } \\
& 0.08 \text { (PvNMT) } \\
& 3.20 \text { (HsNMT) } \\
& 5.82 \text { (LdNMT) }
\end{array}
$$

Fig. 1 NMT affinity profile for 2,3-substituted benzo[b]thiophene 1: aEnzyme apparent $K_{\mathrm{i}}$ values are calculated from the $\mathrm{IC}_{50}$ values using the Cheng-Prusoff equation (see ESI $\uparrow$ ) to enable cross-comparisons. $I C_{50}$ values were the mean of two or more independent determinations. Standard deviation is within $20 \%$ of reported $I C_{50}$. ${ }^{b} \mathrm{HsNMT}$ affinities reported in this work refer to HsNMT1; no significant difference in inhibition was observed between HsNMT1 and HsNMT2 isoforms. ${ }^{18}$

plasmodial NMT, the bicyclic benzo[b]thiophene of 1 was truncated to a monocyclic scaffold (Scheme 1).

The affinity spectrum of compound $\mathbf{5}$ is strikingly distinct from that observed with benzo[b]thiophene 1 (Table 1$)$. The activity against human and both Plasmodium enzymes drops by almost two orders of magnitude, whereas the affinity against LdNMT improves 8-fold, a significant improvement when considering the loss of 4 heavy atoms and attendant reduction in lipophilicity.

Based on this affinity profile and structural analysis of the binding mode of 1 in PvNMT, ${ }^{25}$ it became clear that the monocyclic thiophene scaffold was unlikely to be a suitable chemotype for Plasmodium NMT inhibitors. However, 5 displays a very promising profile as a LdNMT inhibitor with excellent selectivity and LE, as well as sub- $\mu \mathrm{M}$ enzyme affinity. 5 was selected for further development, with the aim of discovering a new lead series for LdNMT inhibitors.

Although heterocycles are often preferred as bioisosteres for a phenyl ring, thiophene has been associated with cytochrome P450 inhibition and generally low "developability" during optimisation. ${ }^{26}$ Replacing the thiophene with a phenyl was deemed preferable, and provided a cost-effective synthetic route and versatility to variations around the scaffold. Pleasingly, this modification from thiophene to phenyl produced a further 3-fold improvement in enzyme affinity, whilst maintaining physicochemical properties and selectivity (Table 1). Interestingly, compounds with a phenyl scaffold proved amenable for development into high affinity Plasmodium NMT inhibitors - this work shall be reported elsewhere. ${ }^{27}$

Compound 6 has a promising affinity profile, but further development of the series would require removal of the

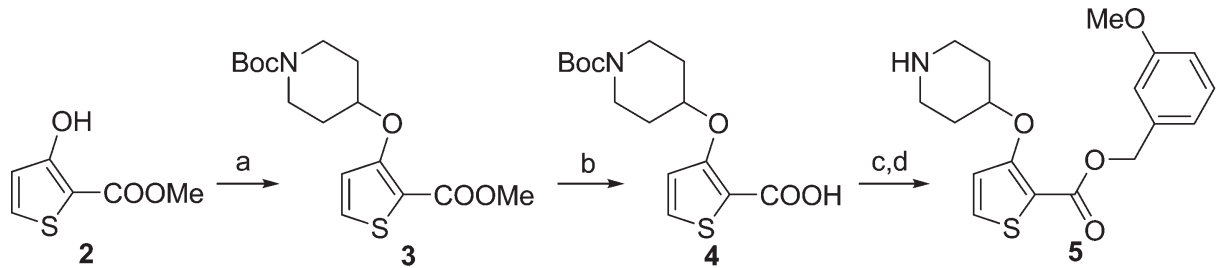

Scheme 1 Synthesis of truncated inhibitor 5. Reagents and conditions: (a) 1-Boc-4-piperidinol, DIAD, PPh $3 . \mathrm{THF}, \mathrm{rt}, 4 \mathrm{~h}, 94 \%$; (b) NaOH, MeOH/ $\mathrm{H}_{2} \mathrm{O}, 50{ }^{\circ} \mathrm{C}, 2 \mathrm{~h}, 90 \%$; (c) (3-methoxyphenyl)methanol, EDCl, HOBt, DIPEA, MeCN, rt, 18 h; (d) 10\% TFA in DCM (v/v), rt, 2 h, $9 \%$ over 2 steps.

Table 1 Enzyme affinity and parasitic LE for mono- and bicyclic analogues 1, 5 and 6

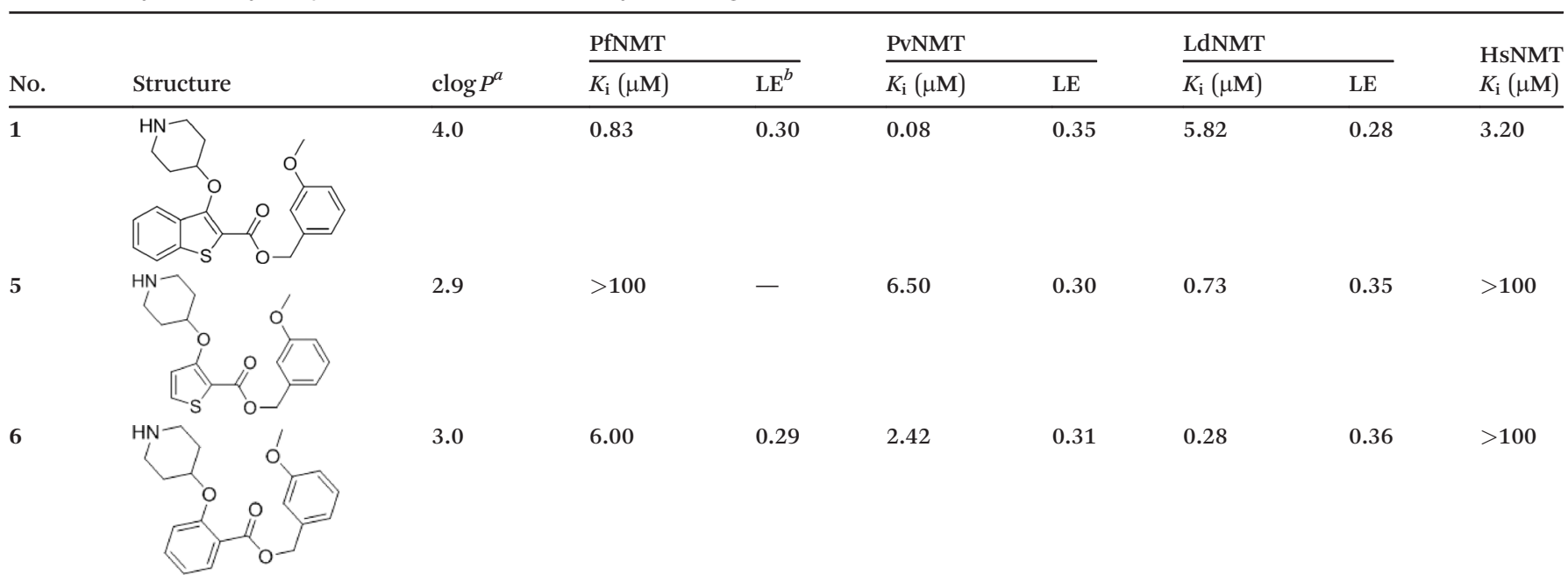

${ }^{a} \operatorname{clog} P$ values calculated with ChemAxon. ${ }^{b} \mathrm{LE}=\left[-\log \left(K_{\mathrm{i}}\right)\right](1.374) /($ no. of heavy atoms $)$. 
potentially labile ester moiety. We have previously reported the successful replacement of the ester moiety with an oxadiazole in related series $;^{24,28}$ the same bioisosteric replacement was implemented in the present series, with the aim of producing a drug-like series for further development. The relatively straightforward synthetic scheme (Scheme 2) enabled facile synthesis of a variety of analogues, thereby allowing investigation of the preferred substituents on this ring system.

Moving from the ester linker in 6 to oxadiazole in 10a produced a $\sim 4$-fold improvement in enzyme affinity alongside removal of the ester moiety. This compound displays measurable affinity against HsNMT but possesses selectivity of 20fold in favour of the parasite enzyme, constituting a highly promising scaffold from which to investigate substituent effects on the phenyl ring (Table 2).

Comparison of 10c, $10 \mathrm{~d}$ and $10 \mathrm{i}$ shows the effect of scanning a methyl substituent around the 3-, 4- and 5-positions of the scaffold. In all cases this variation is less active than the unsubstituted parent 10a, although among these three analogues the 5-substituted $10 \mathrm{i}$ displays the highest affinity.

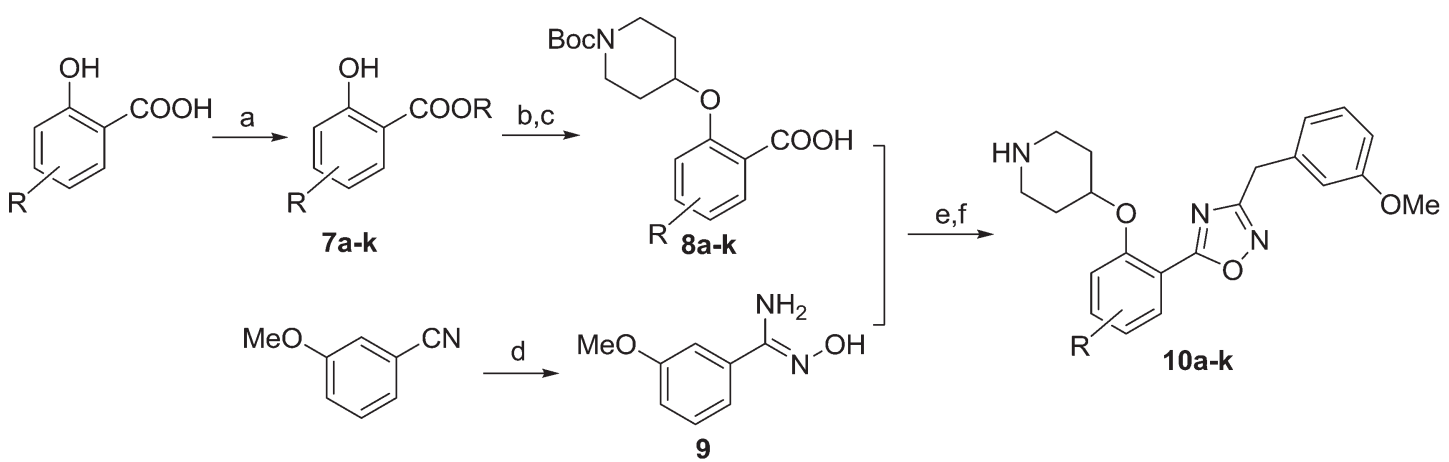

Scheme 2 Synthesis of oxadiazole derivatives 10a-k. Reagents: (a) $\mathrm{BnBr}, \mathrm{K}_{2} \mathrm{CO}_{3}, \mathrm{DMF}, \mathrm{rt}, 2 \mathrm{~h}, 72-89 \%$; (b) DIAD, PPh, $\mathrm{N}-\mathrm{Boc}-4-\mathrm{OH}$ piperidine, THF, rt, 4 h; (c) $\mathrm{NaOH}, \mathrm{MeOH} / \mathrm{H}_{2} \mathrm{O}, 50{ }^{\circ} \mathrm{C}, 2 \mathrm{~h}, 80-95 \%$ over two steps; (d) $\mathrm{NH}_{2} \mathrm{OH}, \mathrm{EtOH}, 80{ }^{\circ} \mathrm{C}, 6 \mathrm{~h}, 98 \%$; (e) i). EDCl, $\mathrm{HOBt}, 9, \mathrm{DIPEA}, \mathrm{CH}{ }_{3} \mathrm{CN}, \mathrm{rt}$, 4 h; ii). $0.5 \mathrm{~N} \mathrm{NaOH}$, rt, 0.5 h; (f) 10\% TFA in DCM, rt, 2 h, 20-60\% over 2 steps.

Table 2 Enzyme affinity and cellular potency for inhibitors based on a phenyl scaffold

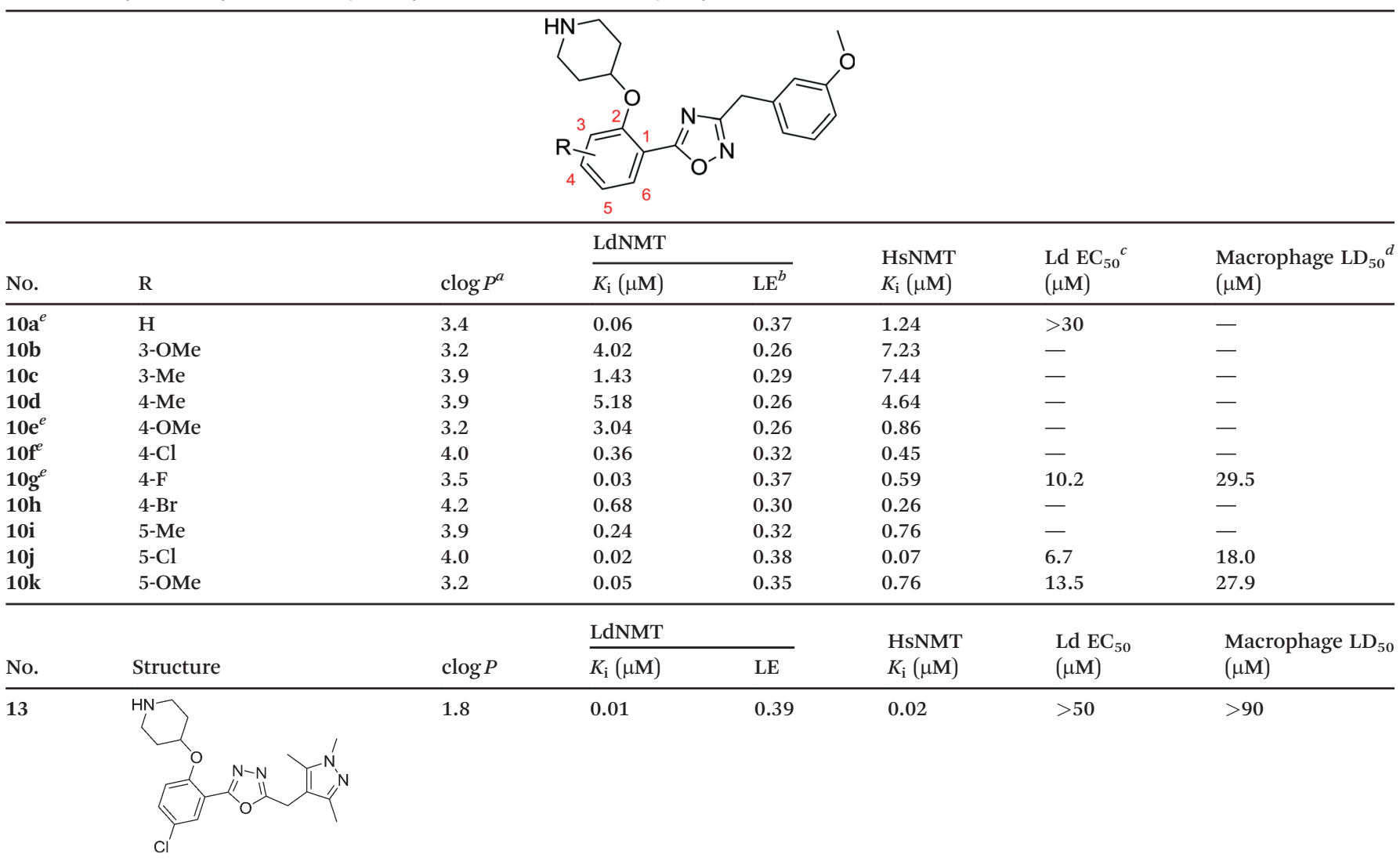

${ }^{a} \operatorname{cog} P$ values calculated with ChemAxon. ${ }^{b} \mathrm{LE}=[-\log (\mathrm{Ki})] \times 1.374 /\left(\right.$ no. of heavy atoms). ${ }^{c}$ Ex vivo L. donovani amastigotes. ${ }^{19}{ }^{d} \mathrm{LD}_{50}$ is an indication of general toxicity of a compound. ${ }^{e}$ This compound is described in Yu et $a l^{27}$ 
This pattern repeats when comparing the methoxysubstituted 10b, 10e and 10k, indicating that the 5-position is preferred for substitution. 5-OMe (10k) provides improved potency over 5 -Me (10i), and this trend continues on moving to the 5 -Cl substituent, identifying $\mathbf{1 0 j}$ as a high affinity LdNMT inhibitor with a $K_{\mathrm{i}}$ of $0.02 \mu \mathrm{M}$.

The binding mode of $\mathbf{1 0 j}$ to Leishmania major NMT $(98 \%$ sequence identity to LdNMT with closely superimposable ligand binding sites) ${ }^{14,29}$ was confirmed by determination of the crystal structure of the ternary complex of LmNMT, myrisotyl-CoA and 10j (PDB ID: 5a27). The inhibitor binds in the expected site adjacent to the enzyme's C-terminal carboxylate to which its piperidine forms an ion pairing interaction. This and other key interactions are conserved in a related series in PvNMT. ${ }^{24}$ Surprisingly, in addition to the observed interactions between the protein and the ligand, the crystal structure revealed a ring-opened derivative of the 1,2,4oxadiazole, rather than the desired heterocycle (see Fig. S1 and $\mathrm{S} 2 \dagger)$. However, proton NMR spectroscopy and high resolution mass spectrometry confirmed that the desired oxadiazole as the synthetic product, furthermore no degradation of 10j was observed when this molecule was incubated at $37{ }^{\circ} \mathrm{C}$ over a wide $\mathrm{pH}$ range ( $\mathrm{pH} 1-10$, data not shown) over a period of 24 hours. This 'ring opening' phenomenon has been observed in related compounds within the program, ${ }^{27}$ although to our knowledge, it has not been reported elsewhere. The origin of the ring-opened species observed in the crystals remains unclear, and further investigation is underway to determine the cause of this discrepancy (such as potential radiation damage). As characterisation of the compounds was consistent with the proposed oxadiazoles, the development of the series was continued.

A selection of compounds with LdNMT $K_{\mathrm{i}}<0.1 \mu \mathrm{M}(10 \mathrm{a}$, $10 \mathrm{~g}, 10 \mathrm{j}, 10 \mathrm{k})$ were tested against ex vivo amastigotes, the clinically-relevant life cycle stage of the parasite, to determine the ability of these molecules to inhibit parasite growth. These molecules displayed a narrow (within 3-fold) therapeutic window against the macrophage host in vitro, and it was unclear whether the observed anti-parasitic activity is a result of NMT inhibition or non-specific toxicity. Given the relatively high $\operatorname{cog} P$ of the tested compounds $(>3.5)$, it was hypothesised that reducing lipophilicity would lead to reduced macrophage toxicity. ${ }^{30}$ We therefore elected to build on our previous success in targeting the S319 pocket through polar heterocyclic replacement of the 3-methoxyphenyl, with the aim of producing a high affinity LdNMT inhibitor with reduced lipophilicity. ${ }^{28}$

Replacing the 1,2,4-oxadiazole with the more polar 1,3,4-regioisomer ${ }^{31}$ and utilising a 1,3,5-trimethylpyrazole motif to replace the hydrophobic methoxyphenyl moiety rendered compound 13 (Scheme 3). Encouragingly, 13 is roughly equipotent to $10 \mathrm{j}$ yet is predicted to be two orders of magnitude less lipophilic, resulting in a highly promising compound. It should be noted that neither 10j nor 13 display significant selectivity for LdNMT over HsNMT, highlighting a target for further development of the series. The crystal structure of 13 bound to LmNMT was solved (PDB ID: 5a28, Fig. 2), and this alternative oxadiazole isomer did not display the ring-opened analogue (Fig. S2†).

The basic piperidine moiety forms a polar interaction with the carboxylate of the C-terminal residue, Leu421 and a water-mediated interaction with Tyr92, mimicking the N-terminus of substrate peptides (Fig. 2B). In addition, the trimethyl pyrazole substituent forms $\pi-\pi$ and polar interactions with Phe90 and Ser330 respectively (Fig. 2C), completing the interactions observed with previous NMT inhibitors. Of particular interest are the interactions around the inhibitor core; a qualitative assessment shows the 5-chlorine inserting into a hydrophobic pocket within the active site, and a lack of space around the 4-position of the scaffold reinforces the lack of activity of the larger substitutions in this position, in the absence of evidence for plasticity in the binding site (Fig. 2A). The rationale for the relative lack of activity of the 3-position substituents is less clear, but may be a result of placing a hydrophobic substituent in a water-filled cavity, or restriction of conformation of the piperidine moiety.

Interestingly, these conserved contacts of 1 and 13 are achieved with remarkably distinct trajectories through the binding site, and a comparison of the binding modes suggests a rationale for the large activity difference between PvNMT and LdNMT for 1 (Fig. 3).

The phenyl ring of the benzothiophene scaffold in 1 occupies a region of the PvNMT binding site that is not occupied by 13 in LmNMT (Fig. 3A), filling a volume occupied by two water molecules in LmNMT (Fig. 3B). Comparison of the residues around this pocket shows some significant

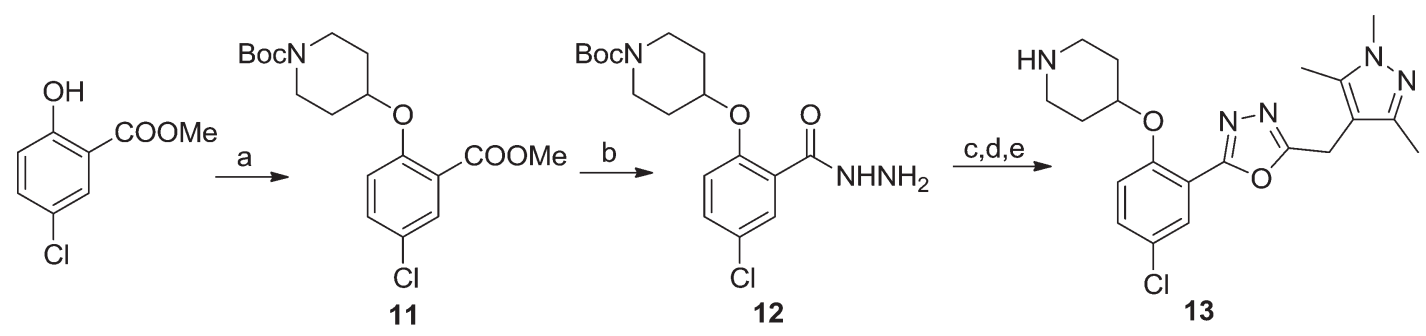

Scheme 3 Synthesis of pyrazole derivative 13. Reagents and conditions: (a) 1-Boc-4-piperidinol, DIAD, PPh 3. THF, rt, 4 h, 46\%; (b) hydrazine monohydrate, EtOH, reflux, $18 \mathrm{~h}$, quantitative; (c) EDCl, HOBt, 2-(trimethyl-1H-pyrazol-4-yl)acetic acid, ${ }^{28}$ DIPEA, THF : DMF (4:1, v/v), rt, 18 h; (d) $\mathrm{TsCl}, 1,2,2,6,6$-pentamethylpiperidine, DCM, rt, 3 h; (e) 10\% TFA in DCM (v/v), rt, 2 h, 40\% over three steps. 


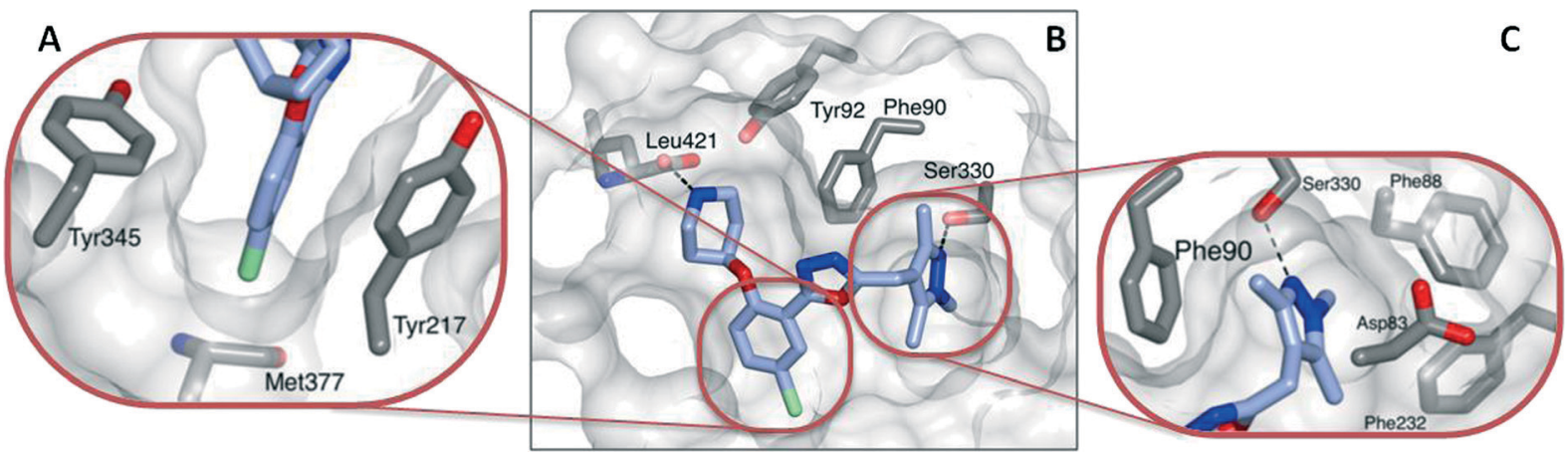

Fig. 2 Crystal structure of 13 (blue) bound to LmNMT (grey). (A) The chloro-substituent is buried within a hydrophobic pocket, and appears to have good shape complementarity with the enzyme active site. (B) In addition to the shape-complementarity of the scaffold, the compound appears to form a similar binding mode to that previously observed. (C) The trimethylpyrazole motif forms both a hydrogen bonding interaction with Ser330 and a hydrophobic stacking interaction with Phe90.
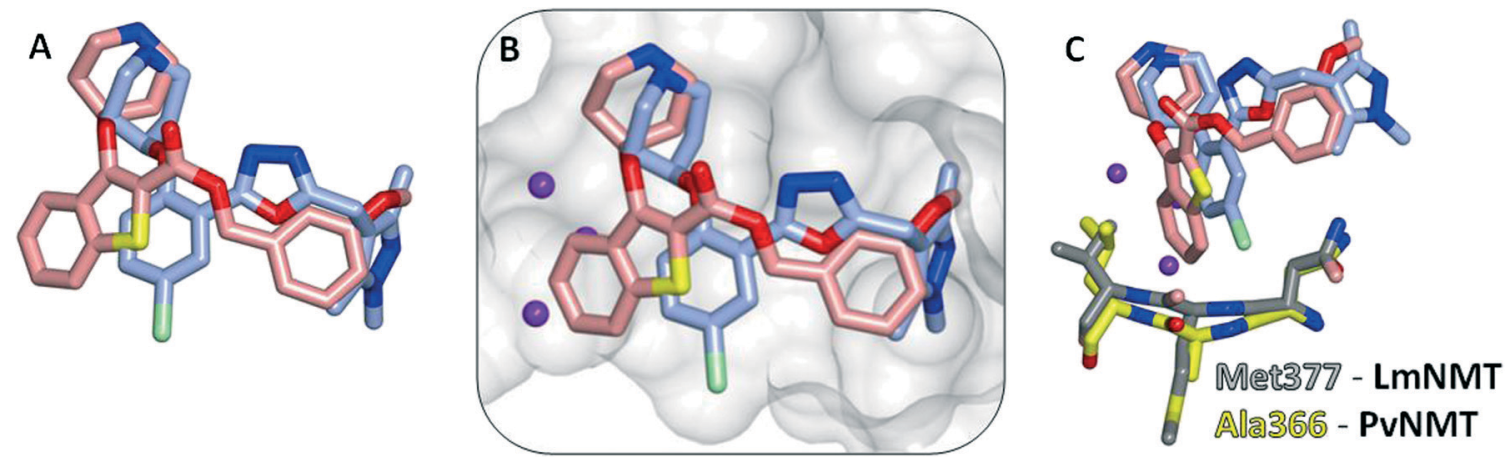

Fig. 3 Comparison of the crystal structure of 1 (pink) bound to PvNMT (yellow) and 13 (blue) bound to LmNMT (grey). (A) Overlay of 1 in PvNMT and 13 in LdNMT, with the enzymes removed for clarity; the phenyl ring of the benzo[b]thiophene scaffold protrudes in the direction of potential 3- and 4-substitutions of the phenyl series. (B) The surface of the active site of LmNMT (grey) shows a constrained, water filled back pocket in the same position as that occupied by the benzo[b]thiophene. (C) Alanine to methionine substitution in LmNMT results in a more constrained pocket and a potential clash with the bulkier benzothiophene scaffold.

differences, the largest of which is Ala366 in PvNMT substituted for Met377 in LmNMT (Fig. 3C). This shifts the protein backbone, and likely results in a more restricted pocket in LmNMT relative to PvNMT. The potential clash between the LmNMT backbone and the benzothiophene core of compound 1 provides a rationale for the reduced affinity of this molecule for LdNMT compared to PvNMT, and also an explanation for the activity loss in molecules carrying 4substituents larger than fluorine (10d-f, 10h vs. 10a, Table 2).

Compound 13 was tested against both macrophages and Ld amastigotes. Pleasingly, 13 did not display any macrophage toxicity at the top concentration tested $(90 \mu \mathrm{M})$, reinforcing the earlier hypothesis that reducing macrophage toxicity can be achieved by reducing lipophilicity. However, this molecule showed no observed effect against ex vivo amastigotes up to $50 \mu \mathrm{M}$.

\section{Conclusion}

In summary, commencing from a member of a previously described series of PfNMT inhibitors, compound 5 was discovered as a highly ligand efficient inhibitor of LdNMT, and subsequent modification of the scaffold yielded the 5-chlorophenyl moiety as the optimum core. However, the compounds showed weak activity when tested against $e x$ vivo amastigotes, and this appeared to correlate with macrophage toxicity; reduction of lipophilicity led to the discovery of compound 13, a high affinity LdNMT inhibitor. The binding mode and selectivity could be rationalised by crystallography, and comparisons to previous structures. Whilst this molecule displayed no macrophage toxicity at all concentrations tested, it also failed to inhibit $L$. donovani amastigotes; the reasons for this lack of cellular activity remain unclear, but it is possible that this may be resolved through further optimisation of the amine basicity and lipophilicity balance in this series. Although NMT has great potential as a drug target in Leishmania parasites, these organisms are notoriously robust, ${ }^{6}$ and the physicochemical properties required for optimal engagement of intracellular parasite drug targets in the intramacrophage amastigote stage remain poorly defined. Further investigation of the influences of the physicochemical properties of this series is currently underway, alongside the introduction of new NMT inhibitor chemotypes ${ }^{18}$ with the aim of chemically validating NMT as a drug target in Leishmania donovani. 


\section{Notes}

The authors declare no competing financial interest.

\section{Acknowledgements}

This work was supported by the Wellcome Trust (grant no.: 087792), the Medical Research Council (grant no.: 0900278 and U117532067) and the EU F97 project MALSIG (HEALTHF3-2009-223044). The authors are grateful to Andrew Bell and Jennie Hutton for valuable discussions, Shirley Roberts for crystal handling and Johan Turkenburg for X-ray data processing. We also acknowledge the Diamond Light Source for synchrotron facilities.

\section{References}

1 R. D. Pearson and A. de Queiroz Sousa, Clin. Infect. Dis., 1996, 22, 1-11.

2 P. Desjeux, Comp. Immunol. Microbiol. Infect. Dis., 2004, 27, 305-318.

3 F. Chappuis, S. Sundar, A. Hailu, H. Ghalib, S. Rijal, R. W. Peeling, J. Alvar and M. Boelaert, Nat. Rev. Microbiol., 2007, 5, 873-882.

4 WHO Leishmaniasis Programme, http://www.who.int/ leishmaniasis/en/itle, 2010.

5 S. L. Croft, S. Sundar and A. H. Fairlamb, Clin. Microbiol. Rev., 2006, 19, 111-126.

6 P. Escobar, S. Matu, C. Marques and S. L. Croft, Acta Trop., 2002, 81, 151-157.

7 M. D. Resh, Trends Mol. Med., 2012, 18, 206-214.

8 E. W. Tate, K. A. Kalesh, T. Lanyon-Hogg, E. M. Storck and E. Thinon, Curr. Opin. Chem. Biol., 2014, 24C, 48-57.

9 M. H. Wright, W. P. Heal, D. J. Mann and E. W. Tate, J. Chem. Biol., 2010, 3, 19-35.

10 E. Thinon, R. A. Serwa, M. Broncel, J. A. Brannigan, U. Brassat, M. H. Wright, W. P. Heal, A. J. Wilkinson, D. J. Mann and E. W. Tate, Nat. Commun., 2014, 5, 4919.

11 K. Kawasaki, M. Masubuchi, K. Morikami, S. Sogabe, T. Aoyama, H. Ebiike, S. Niizuma, M. Hayase, T. Fujii, K. Sakata, H. Shindoh, Y. Shiratori, Y. Aoki, T. Ohtsuka and N. Shimma, Bioorg. Med. Chem. Lett., 2003, 13, 87-91.

12 P. Pino, S. Sebastian, E. A. Kim, E. Bush, M. Brochet, K. Volkmann, E. Kozlowski, M. Llinás, O. Billker and D. Soldati-Favre, Cell Host Microbe, 2012, 12, 824-834.

13 E. W. Tate, A. S. Bell, M. D. Rackham and M. H. Wright, Parasitology, 2014, 141, 37-49.

14 J. A. Frearson, S. Brand, S. P. McElroy, L. A. T. Cleghorn, O. Smid, L. Stojanovski, H. P. Price, M. L. S. Guther, L. S. Torrie, D. A. Robinson, I. Hallyburton, C. P. Mpamhanga, J. A. Brannigan, A. J. Wilkinson, M. Hodgkinson, R. Hui, W. Qiu, O. G. Raimi, D. M. F. van Aalten, R. Brenk, I. H. Gilbert,
K. D. Read, A. H. Fairlamb, M. A. J. Ferguson, D. F. Smith and P. G. Wyatt, Nature, 2010, 464, 728-732.

15 M. H. Wright, B. Clough, M. D. Rackham, K. Rangachari, J. A. Brannigan, M. Grainger, D. K. Moss, A. R. Bottrill, W. P. Heal, M. Broncel, R. A. Serwa, D. Brady, D. J. Mann, R. J. Leatherbarrow, R. Tewari, A. J. Wilkinson, A. A. Holder and E. W. Tate, Nat. Chem., 2014, 6, 112-121.

16 H. P. Price, M. R. Menon, C. Panethymitaki, D. Goulding, P. G. McKean and D. F. Smith, J. Biol. Chem., 2003, 278, 7206-7214.

17 M. H. Wright, D. Paape, E. M. Storck, R. A. Serwa, D. F. Smith and E. W. Tate, Chem. Biol., 2015, 22, 342-354.

18 A. S. Bell, J. E. Mills, G. P. Williams, J. A. Brannigan, A. J. Wilkinson, T. Parkinson, R. J. Leatherbarrow, E. W. Tate, A. A. Holder and D. F. Smith, PLoS Neglected Trop. Dis., 2012, 6, e1625.

19 D. Paape, A. S. Bell, W. P. Heal, J. A. Hutton, R. J. Leatherbarrow, E. W. Tate and D. F. Smith, PLoS Neglected Trop. Dis., 2014, 8, e3363.

20 J. A. Hutton, V. Goncalves, J. A. Brannigan, D. Paape, M. H. Wright, T. M. Waugh, S. M. Roberts, A. S. Bell, A. J. Wilkinson, D. F. Smith, R. J. Leatherbarrow and E. W. Tate, J. Med. Chem., 2014, 57, 8664-8670.

21 J. A. Brannigan, S. M. Roberts, A. S. Bell, J. A. Hutton, M. R. Hodgkinson, E. W. Tate, R. J. Leatherbarrow, D. F. Smith and A. J. Wilkinson, IUCrJ, 2014, 1, 250-260.

22 V. Goncalves, J. A. Brannigan, D. Whalley, K. H. Ansell, B. Saxty, A. A. Holder, A. J. Wilkinson, E. W. Tate and R. J. Leatherbarrow, J. Med. Chem., 2012, 55, 3578-3582.

23 T. O. Olaleye, J. A. Brannigan, S. M. Roberts, R. J. Leatherbarrow, A. J. Wilkinson and E. W. Tate, Org. Biomol. Chem., 2014, 12, 8132-8137.

24 Z. Yu, J. A. Brannigan, D. K. Moss, A. M. Brzozowski, A. J. Wilkinson, A. A. Holder, E. W. Tate and R. J. Leatherbarrow, J. Med. Chem., 2012, 55, 8879-8890.

25 M. D. Rackham, J. A. Brannigan, D. K. Moss, Z. Yu, A. J. Wilkinson, A. A. Holder, E. W. Tate and R. J. Leatherbarrow, J. Med. Chem., 2013, 56, 371-375.

26 T. J. Ritchie, S. J. F. Macdonald, S. Peace, S. D. Pickett and C. N. Luscombe, Med. Chem. Commun., 2012, 3, 1062-1069.

27 Z. Yu, J. A. Brannigan, K. Rangachari, W. P. Heal, A. J. Wilkinson, A. A. Holder, R. J. Leatherbarrow and E. W. Tate, Med. Chem. Commun., Submitted.

28 M. D. Rackham, J. A. Brannigan, K. Rangachari, S. Meister, A. J. Wilkinson, A. A. Holder, R. J. Leatherbarrow and E. W. Tate, J. Med. Chem., 2014, 57, 2773-2788.

29 J. A. Brannigan, B. A. Smith, Z. Yu, A. M. Brzozowski, M. R. Hodgkinson, A. Maroof, H. P. Price, F. Meier, R. J. Leatherbarrow, E. W. Tate, D. F. Smith and A. J. Wilkinson, J. Mol. Biol., 2010, 396, 985-999.

30 P. D. Leeson and B. Springthorpe, Nat. Rev. Drug Discovery, 2007, 6, 881-890.

31 J. Boström, A. Hogner, A. Llinàs, E. Wellner and A. T. Plowright, J. Med. Chem., 2012, 55, 1817-1830. 